\title{
Biochar for Soil Fertility and Natural Carbon Sequestration
}

\author{
Biochar is charcoal (similar to chars generated by forest fires) that is made for incorporation into soils to \\ increase soil fertility while providing natural carbon sequestration. The incorporation of biochar into soils can \\ preserve and enrich soils and also slow the rate at which climate change is affecting our planet. Studies on \\ biochar, such as those cited in this Fact Sheet, are applicable to both fire science and soil science.
}

When plant materials - such as wood, bark, corn stalks, switchgrass, and the like, which scientists generically term biomass - are heated in a low- or no-oxygen atmosphere (this process is termed pyrolysis), the resulting product is called biochar. For example, pyrolysis of wood yields a familiar biochar: charcoal, once the staple fuel for backyard cookouts.

Carbon in the form of carbon dioxide $\left(\mathrm{CO}_{2}\right)$, which is released into the atmosphere when carbon-based fuels are burned and when biomass decays, is a major contributor to global climate change. For this reason, carbon sequestration (the process of capturing $\mathrm{CO}_{2}$ before it escapes into the atmosphere) has the potential to slow global warming. Increasingly, scientists are looking at biochars as additives that can simultaneously enrich soil and sequester carbon that would otherwise be released into the atmosphere if biomass were simply left aboveground to decay.

Plant biomass is primarily a mixture of cellulose and lignin with small amounts of inorganic material called ash. Waste biomass such as beetle-killed trees or agricultural residues can be put to beneficial use by conversion to biochar.

The pyrolysis of plant biomass to generate biochar converts much of the aliphatic carbon (which contains no carbon ring structures) to a very stable form of aromatic carbon (which contains rings made of carbon, like graphite) which is stable in soils for hundreds of years. The term biochar has been adopted to mean char produced from waste biomass for the purpose of land application.

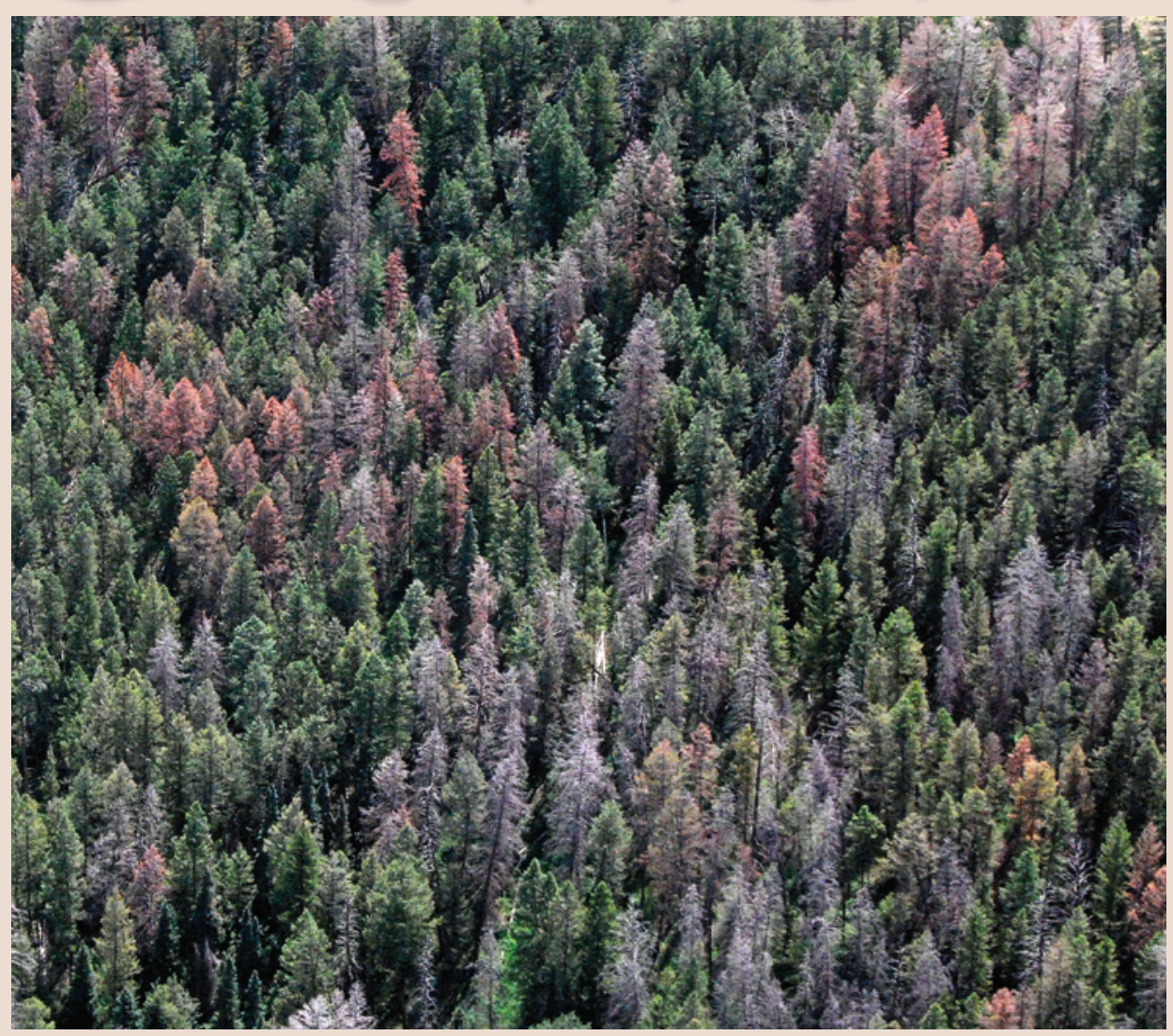

Douglas-fir mortality caused by Douglas-fir beetle in the Uncompahgre National Forest near Telluride, Colorado. Photograph taken by Justin Backsen of the U.S. Forest Service.

The application of biochar to soil has been proposed as a way to store carbon in the soil for longer periods than if biomass was left to decay. Besides increasing the storage of carbon in soils, the application of biochar to soil can improve soil fertility and increase crop production. Since biochar is different from better-known, activated charcoal, information is needed on the effects of its formation conditions on both its physical and chemical properties before farmers begin to use it for large-scale incorporation into soils.

One of a nation's most important assets is the fertility of its soil. The cultivation of soil causes a steady decrease in the soil's organic content and fertility. This steady decrease in fertility is compensated for by addition of fertilizers, but the decrease in organic content is a more permanent problem. Incorporation of biochar can slow or reverse this trend. 


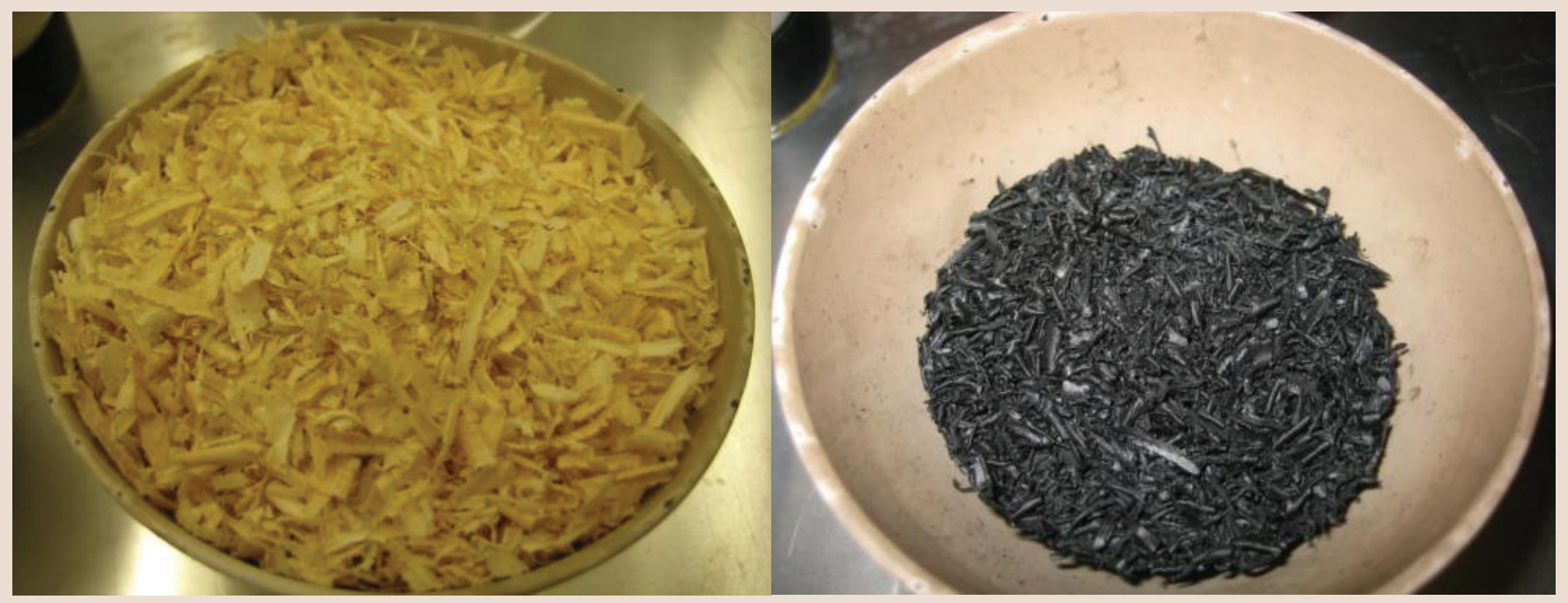

Pine shavings, before and after charring.

As a means of predicting biochar characteristics, the U.S. Geological Survey (USGS) made biochar by charring a variety of feedstocks including purified plant components (such as cellulose and lignin) and plant materials (such as wood, bark, and switchgrass) under a nitrogen atmosphere at temperatures ranging from $200^{\circ} \mathrm{C}$ to $500^{\circ} \mathrm{C}$ (about $400^{\circ} \mathrm{F}$ to $900^{\circ} \mathrm{F}$ ) for times ranging from $1 \mathrm{hr}$ (hour) to $168 \mathrm{hr}$. Changes in composition were examined by mass loss, elemental composition (carbon, hydrogen, and oxygen), acid-functional-group content, and infrared and ${ }^{13} \mathrm{C}$ Nuclear Magnetic Resonance (NMR) spectroscopy (Rutherford and others, 2008). Changes in structure were examined by porosity and surface area (Rutherford and others, 2004).

The USGS has obtained bulk quantities of biochar reference material from two gasification processes which produce biochar as a byproduct. The reference materials were homogenized and split to ensure uniformity. The uniformity of these materials was verified by subsampling and subsequent chemical and physical analyses. These two reference chars provide a consistent material to the scientific research community for comparison of analytical techniques between laboratories or as a basis for comparison of characteristics of one type of char to another.

The two main families of carbon compounds are aliphatic and aromatic. Comparison of mass loss, combined with ${ }^{13} \mathrm{C}$ NMR spectroscopy, showed that the initial rapid loss of material during pyrolysis is due to the loss of aliphatic components, which are either vaporized or converted to aromatic carbon early in the charring process. Aromatic carbon is a more stable form than aliphatic carbon.

The biochars were also analyzed for acid functional group content. Acid functional groups are related to cationexchange capacity, which is important to soil fertility, as it assists in the retention of nutrients (nitrogen and phosphorus) and cations (like potassium) in soil. In the USGS experiments, the highest concentrations of acid functional groups developed at low charring temperatures $\left(250^{\circ} \mathrm{C}\right)$ and long charring times (greater than $72 \mathrm{hr}$ ). Cellulose appears to be the primary source of the acid functional groups in the biochar.

Surface area and porosity of the biochar plays a significant role in soil fertility. In contrast to the optimum conditions for the formation of the acid functional groups, more intense charring conditions (higher temperatures and longer charring times) were required for the formation of porosity and surface area in the biochar.

The effect of formation conditions and feedstock on physical and chemical characteristics of the resultant char will be important if biochar is to be successfully incorporated into agricultural soils. Using this information provided by USGS, formation conditions and feedstocks may be tailored to optimize desirable characteristics (such as acid functional group content) and to minimize less-desirable characteristics (such as degradability or mass loss). The results generated by USGS provide insight into optimum formation conditions for chars with the best properties to simultaneously enhance soil fertility and provide longterm carbon sequestration.

\section{References Cited}

Rutherford, D.W., Wershaw, R.L., and Reeves, J.B., III, 2008, Development of acid functional groups and lactones during the thermal degradation of wood and wood components: U.S. Geological Survey Scientific Investigations Report 2007-5013, 43 p.

(Also available at http://pubs.usgs.gov/ sir/2007/5013)

Rutherford, D.W., Wershaw, R.L., and Cox, L.G., 2004, Changes in composition and porosity occurring during the thermal degradation of wood and wood components: U.S. Geological Survey Scientific Investigations Report 2004-5292, 78 p. (Also online at http://pubs.usgs.gov/sir/2004/5292/.)

—Colleen E. Rostad (cerostad@usgs.gov) David W. Rutherford (dwruther@usgs.gov)

For more information, contact:

Chief Scientist, Hydrology

Branch of Hydrologic Research

U.S. Geological Survey

409 National Center

12201 Sunrise Valley Drive

Reston, VA 20192 